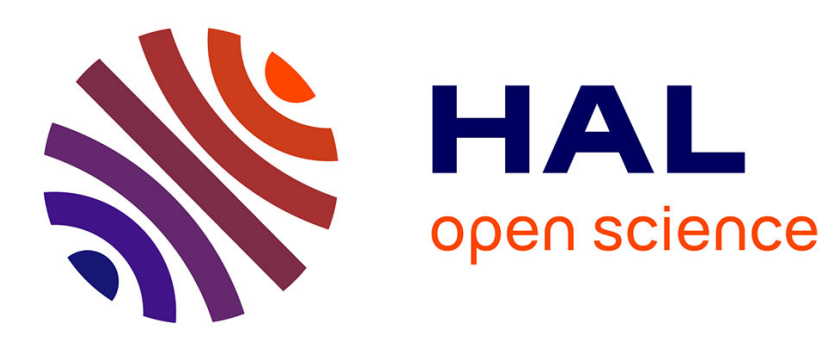

\title{
Boundary conditions for the electron kinetic equation using expansion techniques
}

\author{
M. M. Becker, G. K. Grubert, D. Loffhagen
}

\section{To cite this version:}

M. M. Becker, G. K. Grubert, D. Loffhagen. Boundary conditions for the electron kinetic equation using expansion techniques. European Physical Journal: Applied Physics, 2010, 51 (1), 10.1051/epjap/2010073 . hal-00601164

\section{HAL Id: hal-00601164 \\ https://hal.science/hal-00601164}

Submitted on 17 Jun 2011

HAL is a multi-disciplinary open access archive for the deposit and dissemination of scientific research documents, whether they are published or not. The documents may come from teaching and research institutions in France or abroad, or from public or private research centers.
L'archive ouverte pluridisciplinaire HAL, est destinée au dépôt et à la diffusion de documents scientifiques de niveau recherche, publiés ou non, émanant des établissements d'enseignement et de recherche français ou étrangers, des laboratoires publics ou privés. 


\title{
Boundary conditions for the electron kinetic equation using
}

\section{expansion techniques}

\author{
M.M. Becker ${ }^{1}$, G.K. Grubert ${ }^{2}$, and D. Loffhagen ${ }^{1}$ \\ 1 INP Greifswald, Felix-Hausdorff-Str. 2, 17489 Greifswald, Germany \\ 2 University of Greifswald, Felix-Hausdorff-Str. 12, 17489 Greifswald, Germany
}

Received: date / Revised version: date

\begin{abstract}
The numerical solution of partial differential equations requires suitable boundary conditions. Based on a fundamental integral relation for Legendre polynomials, Marshak type boundary conditions are derived that can be applied if an expansion in Legendre polynomials is utilized to solve the spatially inhomogeneous kinetic equation of the electrons in gas discharge plasmas. In particular, it is now possible to relate directly the expansion coefficients of the phase space distribution with macroscopic quantities at the boundaries. That is, a well-defined influx or a reflection of electrons can easily be treated on the microscopic level.
\end{abstract}

PACS. 52.25.Dg Plasma kinetic equations - 51.10.+y Kinetic and transport theory of gases $-52.80 .-\mathrm{s}$ Electric discharges

\section{Introduction}

The electron component determines decisively the behaviour of gas discharge plasmas. For a theoretical description, their kinetic equation has to be treated and different methods for its solution have been developed. On the one hand, there are expansion techniques which have been applied. Depending on the discharge conditions, expansions

Correspondence to: markus.becker@inp-greifswald.de in spherical harmonics or in Legendre polynomials have generally been performed [1-5], where the latter expansion represents a special case of the spherical harmonics expansion requiring e.g. the simplifying assumption that the electric field action and the inhomogeneity of the plasma occur in the same direction [2]. On the other hand, simulation techniques like Monte-Carlo methods [6-11] have been used to determine the kinetic properties of the electrons. 
The treatment of boundary conditions and their representation in terms of measurable properties is essential for the solution of the electron Boltzmann equation and other kinetic approaches to model gas discharge plasmas. When solving the space-dependent kinetic equation of the electrons, different approximations for the electron momentum distribution function (EMDF) have been employed. For instance, relations of the form $[12-15]$

$$
f_{0}(r, U) \sim f_{r}(r, U)
$$

have been adapted for the analysis of the radial inhomogeneous column plasma of glow discharges, in order to describe absorption and reflection of electrons at the wall of the discharge tube within the framework of the two-term approximation of the expansion of the EMDF in spherical harmonics. Here, $f_{0}$ and $f_{r}$ are the isotropic part and the radial contribution to the distribution anisotropy, which depend on the radial position $r$ and the kinetic energy $U$ of the electrons. An alternative condition fixing the radial anisotropy $f_{r}$ at the tube wall by an exponentially decreasing positive function $g(U)$ has been used in $[16,17]$. This condition has been taken to describe a positive current of low-energy electrons to the wall, where the shape of $g(U)$ has been chosen to correspond sufficiently to the shape of the positive part of $f_{r}$ in the inner part of the dc column plasma. However, its physical justification remained open.

In order to analyse the axial behaviour of the electrons in discharge between plane electrodes, relations similar to equation (1) have been employed in [18-21] to characterize the absorption and partial reflection of electrons at the anode. A generalization of this boundary condition at the an- ode for the multiterm approximation of the Legendre polynomial expansion of the EMDF has been reported in [22]. The assumption of complete absorption of electrons at the anode has been made in the kinetic studies of $[23,24]$ using a two-term Legendre polynomial expansion of the EMDF. Boundary conditions at infinity of the solution region for the multiterm analysis of non-hydrodynamic swarms of electrons in situations where both conservative and nonconservative collisions take place have been discussed e.g. in [25]. But it is stated in that paper that these conditions do not represent conditions at any physical boundary.

Conditions characterizing the inflow of electrons from an electron source or from the cathode of a discharge have been represented e.g. in [25-29]. However, a physical motivation for the condition of the emission of electrons from the cathode of the discharge has been given for the first contribution to the distribution anisotropy only.

The present work focuses on discharge conditions at which an expansion of the EMDF in Legendre polynomials can be applied. For the numerical determination of the corresponding expansion coefficients of the EMDF, the choice of suitable boundary conditions plays an important role. Based on an ansatz similar to that proposed by Marshak [30-33] and a fundamental integral relation for Legendre polynomials, explicit expressions for the expansion coefficients have been derived that can be used as boundary conditions and relate macroscopic properties of the electrons with the expansion coefficients at the spatial margins of the discharge volume. In particular, the boundary conditions presented make it possible to accu- 


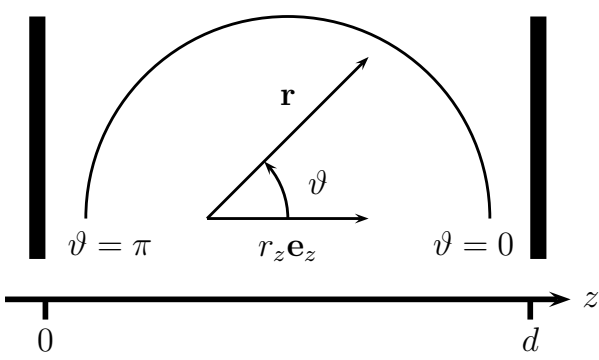

Fig. 1. Axially inhomogeneous discharge geometry.

rately treat physical effects like secondary electron emission, reflection and back-diffusion on the kinetic level for an arbitrary order of the expansion.

In order to demonstrate the superiority of the new boundary conditions, a comparison with commonly used boundary conditions is presented using the example of an abnormal oxygen glow discharge.

\section{Electron kinetic equation}

To describe the electron component in gas discharge plasmas at steady state, the Boltzmann equation

$$
\begin{aligned}
\left(\frac{\mathbf{p}}{m_{e}} \cdot \nabla_{\mathbf{r}}+\mathbf{F}^{\mathrm{ext}} \cdot\right. & \left.\nabla_{\mathbf{p}}\right) \tilde{f}(\mathbf{r}, \mathbf{p}) \\
& =\sum_{a}\left[\mathbb{I}_{a}^{\mathrm{el}}(\tilde{f})+\sum_{i} \mathbb{I}_{i, a}^{\mathrm{in}}(\tilde{f})\right]
\end{aligned}
$$

for the $\operatorname{EMDF} \tilde{f}(\mathbf{r}, \mathbf{p})$ has to be solved. Here, $\mathbf{F}^{\text {ext }}=$ $-e_{0} \mathbf{E} / m_{e}$ is the electric field force acting on the plasma and $\mathbb{I}_{a}^{\mathrm{el}}$ and $\mathbb{I}_{i, a}^{\mathrm{in}}$ are the individual collision integrals for elastic scattering and the $i$ th inelastic collision processes of electrons having the mass $m_{e}$ with gas particles of kind ' $a$ '.

Considering the spatially one-dimensional geometry of discharges between plane electrodes illustrated in Fig. 1, where $\mathbf{E}$ and the plasma inhomogeneity are parallel to the $z$ direction, the EMDF gets the reduced dependence $\tilde{f}\left(z, p, p_{z} / p\right)$ on the space coordinate $z$, the magnitude of the momentum $p$, and the direction cosine $p_{z} / p=$ $\cos \vartheta$. Thus, it can be expanded in Legendre polynomials $P_{n}\left(p_{z} / p\right)[4]$, that is

$$
\tilde{f}\left(z, p, \frac{p_{z}}{p}\right)=\sum_{n=0}^{\infty} \tilde{f}_{n}(z, p) P_{n}\left(\frac{p_{z}}{p}\right) .
$$

The substitution of this expansion up to an arbitrary number $l$ of expansion coefficients $\tilde{f}_{n}(z, p)$ into the kinetic equation (2) and the transformation to the electron kinetic energy $U=p^{2} /\left(2 m_{e}\right)$ finally lead to the hierarchy of partial differential equations [34]

$$
\begin{aligned}
& \frac{n}{2 n-1} U \partial_{z} f_{n-1}(z, U)+\frac{n+1}{2 n+3} U \partial_{z} f_{n+1}(z, U) \\
& -\frac{n}{2 n-1} \frac{e_{0}}{m_{e}} E_{z}\left[U \partial_{U} f_{n-1}(z, U)-\frac{n-1}{2} f_{n-1}(z, U)\right] \\
& -\frac{n+1}{2 n+3} \frac{e_{0}}{m_{e}} E_{z}\left[U \partial_{U} f_{n+1}(z, U)+\frac{n+2}{2} f_{n+1}(z, U)\right] \\
& =\sum_{a} 2 N_{a} \frac{m_{e}}{M_{a}} \partial_{U}\left[U^{2} Q_{a}^{\mathrm{mt}}(U) f_{n}(z, U)\right] \delta_{0 n} \\
& -\sum_{a} U N_{a}\left[Q_{a}^{\mathrm{mt}}(U) \theta(n-1)+\sum_{i} Q_{i, a}^{\mathrm{in}}(U)\right] f_{n}(z, U) \\
& +\sum_{a} N_{a} \sum_{i}\left(U+U_{i, a}^{\mathrm{in}}\right) Q_{i, a}^{\mathrm{in}}\left(U+U_{i, a}^{\mathrm{in}}\right) \\
& \quad \times f_{n}\left(z, U+U_{i, a}^{\mathrm{in}}\right) \delta_{0 n}
\end{aligned}
$$

for the expansion coefficients

$$
f_{n}(z, U)=2 \pi\left(2 m_{e}\right)^{3 / 2} \tilde{f}_{n}(z, p(U))
$$

with $n=0, \ldots, l-1$. Here, $N_{a}$ is the density of the heavy particle of kind ' $a$ ' with the mass $M_{a} . Q_{a}^{\mathrm{mt}}$ and $Q_{i, a}^{\text {in }}$ denote the momentum transfer cross section for elastic collisions and the total cross section for the $i$ th inelastic collision processes with the particle component ' $a$ ', respectively. $U_{i, a}^{\text {in }}$ is the corresponding energy loss of electrons due to the inelastic collision process. In particular, 


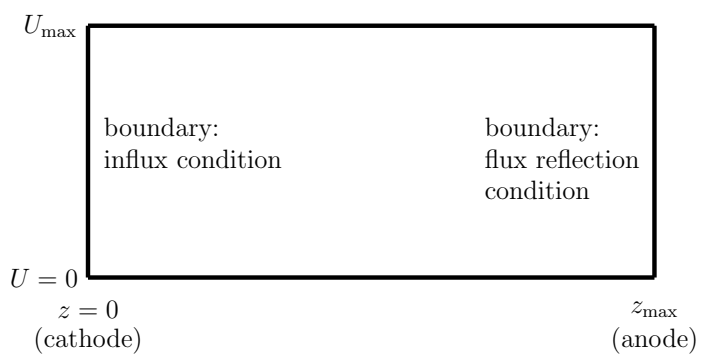

Fig. 2. Natural solution region in $(z, U)$ coordinates.

isotropic scattering of the electrons in inelastic collisions processes has been assumed. The system of partial differential equations (4) can be solved numerically as an initial-boundary value problem provided that appropriate initial values and boundary conditions are given at the spatial margins $z=0$ and $z=z_{\max }$ of the solution region $\left(0 \leq z \leq z_{\max }, 0 \leq U \leq U_{\max }\right)$ represented in Fig. 2. Here, $U_{\max }$ is an appropriate upper energy limit of the kinetic energy above which all components of the EMDF can be assumed to be already negligibly small, i.e., $f_{n}\left(z, U \geq U_{\max }\right)=0$. Such a value $U_{\max }$ can be found approximately, for example, from the solution of the corresponding spatially homogeneous Boltzmann equation.

For the numerical solution of system (4) it is convenient to replace the kinetic energy $U$ by the total energy $\varepsilon=U+W(z)$ with $W(z)=e_{0} \int_{0}^{z} E_{z}\left(z^{\prime}\right) \mathrm{d} z^{\prime}$ being the potential energy of the electrons [35]. After the transformation of Eq. (4) to the $(z, \varepsilon)$ coordinates and the discretization with respect to $z$ and $\varepsilon$ on the centred grid points $\left(z_{i+1 / 2}, \varepsilon_{k+1 / 2}\right)$ with $1 \leq i \leq i_{\max }$ and $1 \leq k \leq k_{\max }$, the numerical solution as initial-boundary value problem takes place by advancing from higher to lower total energies [34] within the transformed solution region rep-

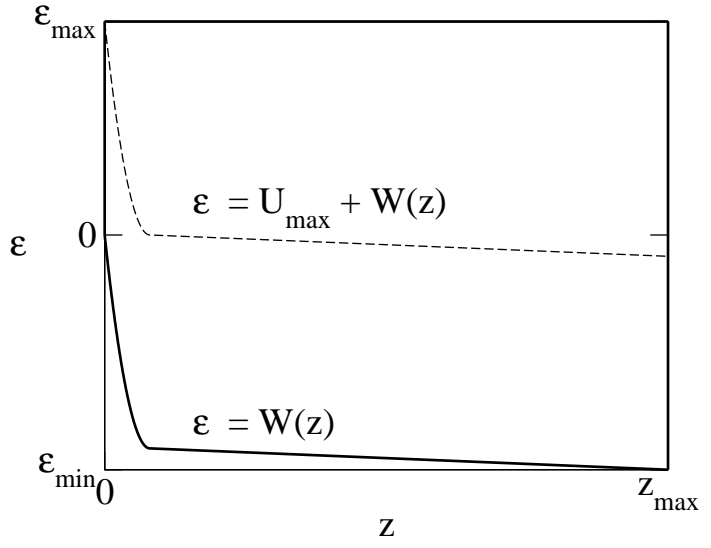

Fig. 3. Solution region in the transformed $(z, \varepsilon)$ space. The dotted line represents the total energy $\varepsilon=U_{\max }+W(z)$.

resented in Fig. 3. The space-dependence of the electric field and of the heavy particle densities are assumed to be given.

The numerical solution starts at $\varepsilon_{\max }$ where all transformed expansion coefficients $\hat{f}_{n}(z, \varepsilon)=f_{n}(z, U(z, \varepsilon))$ are negligibly small [34]. At the boundary $\varepsilon=W(z)$, i.e. at vanishing kinetic energy, the condition $f_{n}(z, U=0)=0$ for all $n \geq 1$ can immediately be derived when analysing system (4) in the limit of zero kinetic energy [35]. For the numerical solution this boundary condition is only required for all odd components.

In the following appropriate conditions at the spatial margins $z=0$ and $z=z_{\max }$ are discussed. Although system (4) is numerically solved after transformation to the total energy space, the boundary conditions are derived for the natural solution region shown in Fig. 2. The corresponding relations derived can easily be transferred to the total energy region. 


\section{Derivation of the boundary conditions}

The system of hierarchy equations (4) represents a set of partial differential equations of first order. That is, the number of necessary boundary conditions equals the the number $l$ of expansion coefficients. Here, $\lfloor l / 2\rfloor$ conditions are applied at $z=0$ to the coefficients $f_{2 n+1}(z, U)$ with an odd index and $\lfloor(l+1) / 2\rfloor$ conditions are applied at $z=z_{\max }$ to the coefficients $f_{2 n}(z, U)$ with an even index.

According to the physical situation considered, suitable boundary conditions have to characterize (i) an influx of electrons leaving the cathode at $z=0$ due to the impact of, e.g., ions, fast neutrals, metastable atoms, or photons impinging onto this electrode and (ii) the absorption or reflection of electrons at the anode.

Following the approach of Marshak [30], the boundary conditions are derived from macroscopic properties by considering the moments of the electron kinetic equation (2) of odd order. In general, the $z$ component of the $k$ th order moments of Eq. (2) is given by [2]

$$
\begin{aligned}
\mathbb{G}_{z, k}(z)= & \frac{1}{2}\left(\frac{2}{m_{e}}\right)^{k / 2} \int_{0}^{\infty} d U U^{(k+1) / 2} \\
& \times \int_{-1}^{1} d x x^{k} f(z, U, x)
\end{aligned}
$$

with $x=p_{z} / p$. Starting from this expression for $k=2 \varrho+1$, the $l$ boundary conditions needed are found from requiring appropriate conditions at both the spatial margins.

\subsection{Influx condition at the cathode}

At the cathode side, a specification of the influx of electrons provides a meaningful boundary condition. The in- flux of electrons leaving the cathode and moving towards the anode can be characterized by the moments

$$
\begin{aligned}
\mathbb{G}_{z, 2 \varrho+1}^{\mathrm{CA}}(0)= & \frac{1}{2}\left(\frac{2}{m_{e}}\right)^{\varrho+1 / 2} \int_{0}^{\infty} d U U^{\varrho+1} \\
& \times \int_{0}^{1} d x x^{2 \varrho+1} f(0, U, x)
\end{aligned}
$$

of the order $2 \varrho+1$ with $\varrho \in \mathbb{N}_{0}$, where the integration of the angular variable $x$ of the momentum space occurs over the half-range [31] as shown in Fig 1. The substitution of $f(0, U, x)$ in Eq. (7) by its $l$-term approximation according to Eq. (3) and the application of the integral relation (29) given in the appendix yield

$$
\begin{aligned}
\mathbb{G}_{z, 2 \varrho+1}^{\mathrm{CA}}(0)= & \frac{(2 \varrho+1) !}{2}\left(\frac{2}{m_{e}}\right)^{\varrho+1 / 2} \int_{0}^{\infty} d U U^{\varrho+1} \\
& \times \sum_{n=0}^{l-1} \frac{f_{n}(0, U)}{(2 \varrho+1-n) ! !(2 \varrho+n+2) ! !} .
\end{aligned}
$$

Note that the expansion in Legendre polynomials is not affected by the discontinuity of the distribution function separating ingoing and outgoing particles because the halfrange of the angular variable of the momentum space is taken into account only.

Applying an appropriate normalized distribution function $f^{S}(U, x)$ at the cathode, e.g., a distribution with a Gaussian-like energy profile and a forward directed or an isotropic inflow of electrons into the half-range, the relation

$$
\begin{array}{r}
\int_{0}^{\infty} d U U^{\varrho+1} \int_{0}^{1} d x x^{2 \varrho+1} f^{S}(U, x) \\
=\int_{0}^{\infty} d U U^{\varrho+1} \sum_{n=0}^{l-1} \frac{(2 \varrho+1) ! f_{n}(0, U)}{(2 \varrho+1-n) ! !(2 \varrho+n+2) ! !}
\end{array}
$$

is obtained by equating expressions (7) and (8) using $f(0, U, x)=f^{S}(U, x)$. Clearly, this equation is fulfilled if 
the sum at the right-hand side of Eq. (9) equals the halfrange integral at the left-hand side of Eq. (9) for all values of $U$, i.e., if the relation

$$
\sum_{n=0}^{l-1} \frac{(2 \varrho+1) ! f_{n}(0, U)}{(2 \varrho+1-n) ! !(2 \varrho+n+2) ! !}=C_{2 \varrho+1}(U)
$$

with

$$
C_{2 \varrho+1}(U)=\int_{0}^{1} d x x^{2 \varrho+1} f^{S}(U, x)
$$

holds. The required boundary conditions at the cathode are finally obtained by splitting Eq. (10) into sums of expansion coefficients with exclusively odd and even indices, respectively. Using definition (28) given in the appendix, the energy-dependent boundary conditions for the expansion coefficients $f_{n}(z, U)$ with odd $n$ reads

$$
\begin{gathered}
\sum_{i=0}^{\varrho} \frac{(2 \varrho+1) ! f_{2 i+1}(0, U)}{(2 \varrho-2 i) ! !(2 \varrho+2 i+3) ! !}=C_{2 \varrho+1}(U) \\
\quad-\sum_{i=0}^{\lfloor(l-1) / 2\rfloor} \frac{(2 \varrho+1) ! f_{2 i}(0, U)}{(2 \varrho-2 i+1) ! !(2 \varrho+2 i+2) ! !}
\end{gathered}
$$

for $\varrho=0, \ldots,\lfloor l / 2\rfloor-1$. Eq. (12) represents relations that allow the determination of $f_{n}(0, U)$ with odd $n$ in dependence on $f_{n}(0, U)$ with even $n$ and an additional term whose energy dependence is explicitly known. For instance, the first contribution $f_{1}(0, U)$ to the distribution anisotropy of the EMDF is given by the relation

$$
\begin{aligned}
f_{1}(0, U)= & 3 C_{1}(U) \\
& -3 \sum_{i=0}^{\lfloor(l-1) / 2\rfloor} \frac{f_{2 i}(0, U)}{(-2 i+1) ! !(2 i+2) ! !}
\end{aligned}
$$

describing the microscopic particle flux of the electrons. The corresponding macroscopic particle flux of electrons is obtained by means of an appropriate energy space averaging of Eq. (13).

In contrast to previous studies assuming vanishing odd expansion coefficients $f_{n}(z, U)$ with $n=3,5, \ldots$ at the cathode $[26,27,34]$, Eq. (12) provides a much better approximation of the boundary condition

$$
f^{l}(0, U, x)=f^{S}(U, x)
$$

where $f^{l}(0, U, x)$ denotes the representation of $f(0, U, x)$ in $l$-term approximation. Notice, that the derivation of boundary conditions that provide a mathematically exact fulfillment of Eq. (14) seems to be impossible when a truncated Legendre polynomial expansion for solving Eq. (2) is used $[32,36]$.

\subsection{Reflection condition at the anode}

At the anode side, relations that describe the reflection or absorption of electrons provide suitable boundary conditions. To characterize partial reflection of electrons at the anode, the flux of electrons can be decomposed into a flux towards the wall and away from it. The latter can be described by the moments

$$
\begin{aligned}
\mathbb{G}_{z, 2 \varrho+1}^{\mathrm{AC}}\left(z_{\max }\right)= & \frac{1}{2}\left(\frac{2}{m_{e}}\right)^{\varrho+1 / 2} \int_{0}^{\infty} d U U^{\varrho+1} \\
& \times \int_{-1}^{0} d x x^{2 \varrho+1} f\left(z_{\max }, U, x\right) .
\end{aligned}
$$

It is related to the flux $\mathbb{G}_{z, 2 \varrho+1}^{\mathrm{CA}}\left(z_{\max }\right)$ towards the anode according to

$$
\mathbb{G}_{z, 2 \varrho+1}^{\mathrm{AC}}\left(z_{\max }\right)=-r \mathbb{G}_{z, 2 \varrho+1}^{\mathrm{CA}}\left(z_{\max }\right)
$$

for arbitrary moments of the order $2 \varrho+1$ with $\varrho \in \mathbb{N}_{0}$. Here, $r \in[0,1[$ is the reflection coefficient of electrons at the anode. Starting from Eq. (16), boundary conditions for the expansion coefficients with an even index can be 
derived by means of a procedure similar to that described in section 3.1 using the relation (32) given in the appendix. The energy-dependent conditions at $z=z_{\max }$ read

$$
\begin{aligned}
& \frac{1-r}{1+r} \sum_{i=0}^{\lfloor(l-1) / 2\rfloor} \frac{(2 \varrho+1) ! f_{2 i}\left(z_{\max }, U\right)}{(2 \varrho-2 i+1) ! !(2 \varrho+2 i+2) ! !} \\
& =\sum_{i=0}^{\varrho} \frac{(2 \varrho+1) ! f_{2 i+1}\left(z_{\max }, U\right)}{(2 \varrho-2 i) ! !(2 \varrho+2 i+3) ! !}
\end{aligned}
$$

for $\varrho=0, \ldots,\lfloor(l-1) / 2\rfloor$. These equations relate a sum of expansion coefficients $f_{n}\left(z_{\max }, U\right)$ with only even indices $n$ to a sum of expansion coefficients with only odd indices $n$. They provide an approximation of the boundary condition [32]

$$
f^{l}\left(z_{\max }, U, x\right) \stackrel{x \leq 0}{=} r f^{l}\left(z_{\max }, U,-x\right)
$$

where $f^{l}\left(z_{\max }, U, x\right)$ denotes the representation of $f\left(z_{\max }, U, x\right)$ in $l$-term approximation. Eq. (17) is in agreement with the relation (21) of Ref. [22], which was also justified by comparison with Monte Carlo simulations in that paper.

In the limit of pure diffusion, i.e. when $E_{z}=0$, the relation

$$
f_{1}(z, U)=-\lambda_{\mathrm{m}}(z, U)\left(\partial_{z} f_{0}(z, U)+\frac{2}{5} \partial_{z} f_{2}(z, U)\right)
$$

with the mean free path of the electrons

$$
\lambda_{\mathrm{m}}(z, U)=\frac{1}{\sum_{a} N_{a}(z)\left(Q_{a}^{\mathrm{mt}}(U)+\sum_{i} Q_{i, a}^{\mathrm{in}}(U)\right)}
$$

results from (4) for $n=1$. The combination of (17) for $\rho=0$ and (19) at $z=z_{\max }$ and the subsequent integration over the energy space finally yield in the limit of the twoterm approximation the equation

$$
\left.\frac{\nabla_{z} n_{e}(z)}{n_{e}(z)}\right|_{z=z_{\max }}=-\frac{3}{2 \lambda_{\mathrm{m}}} \frac{1-r}{1+r}
$$

for the electron density $n_{e}(z)=\int_{0}^{\infty} \sqrt{U} f_{0}(z, U) \mathrm{d} U$, where an average electron collisional mean free path $\lambda_{\mathrm{m}}$ has been used. Equation (21) is in agreement with the formula for diffusion calculations involving wall reflection and low density derived in [37].

\subsection{Comparison of boundary conditions at the cathode}

In this section, cathode-sided boundary conditions are evaluated by comparison with the distribution function $f^{S}(U, x)$ assumed for the electrons emitted from the cathode. In order to illustrate the effect of boundary conditions, an abnormal oxygen glow discharge was investigated by numerically solving the set of equations (4) for given spatial profiles of the electric field $E_{z}$ and the heavy particle densities $N_{a}$. Details of the discharge parameters and the underlying cross sections can be found in Ref. [34]. In particular, the set of electron-heavy particle collisions in the plasma includes both conservative processes, namely elastic collisions, vibrational and electronic excitation as well as dissociation, and non-conservative electron processes, i.e. ionization and dissociative electron attachment.

The numerical solution of the equation system (4) was obtained by an approximation with 20 terms in the Legendre polynomial expansion (3). To solve system (4), the boundary conditions (12) were used for each odd expansion coefficient at the cathode including the angular dependence of the electron influx. The required source function $f^{S}(U, x)$ at the cathode was chosen as

$$
f^{S}(U, x)=U \exp \left(-\left(\frac{U-U_{w}}{U_{c}}\right)^{2}\right)
$$


with the energetic width and centre of the distribution $U_{w}=5 \mathrm{eV}$ and $U_{c}=2 \mathrm{eV}$, respectively. This source function corresponds to an isotropic influx of electrons into the half-range with a Gaussian-like energy profile. The consideration of an isotropic inscattering is motivated by its usage in a lot of theoretical plasma investigations, see, e.g., Ref. [7]. The corresponding relation for the electron flux determined by the first anisotropic component of the EMDF reads

$$
\begin{aligned}
f_{1}(0, U)= & \frac{3}{2} U \exp \left(-\left(\frac{U-U_{w}}{U_{c}}\right)^{2}\right) \\
& -3 \sum_{i=0}^{\lfloor(l-1) / 2\rfloor} \frac{f_{2 i}(0, U)}{(-2 i+1) ! !(2 i+2) ! !} .
\end{aligned}
$$

Furthermore, the conditions (17) were employed at the anode side using $r=0.36$.

In the literature $[26,27,34]$, the inscattering profile (22) is commonly applied to $f_{1}(0, U)$ only, i.e.,

$$
f_{1}(0, U)=U \exp \left(-\left(\frac{U-U_{w}}{U_{c}}\right)^{2}\right)
$$

and all further expansion coefficients $f_{n}(0, U)$ with odd $n \geq 3$ are set to zero. Higher order corrections by $f_{n}(0, U)$ with even $n$ as present in Eq. (23) are completely neglected.

In Fig. 4, numerical results for the distribution function $f(z=0, U, x)$ at the cathode, normalized to the maximum of the source function $f^{S}(U, x)$, are compared with $f^{S}(U, x)$ at $x=1 / 2$. For the numerical computations, the boundary conditions (12) and (24), respectively, have been used. The comparison shows that the results based on the new boundary conditions (12) match the distribution function $f^{S}(U, x=1 / 2)$. In contrast to that, appreciable

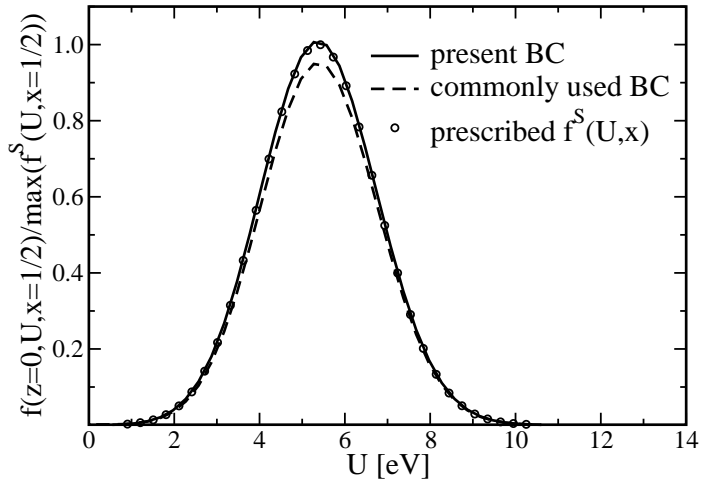

Fig. 4. Comparison of the distribution functions $f(0, U, x)$ obtained by a 20 -term approximation using the boundary conditions (BC) according to (12) and (24) with the inscattering profile (22) of the electrons at the angular variable $x=1 / 2$.

differences between the results based on the commonly used boundary condition (24) neglecting the contributions of higher odd expansion coefficients and the analytically given function $f^{S}(U, x=1 / 2)$ are found. That is, expansion coefficients of higher order have to be included if the electron kinetic equation (2) is solved.

Notice, that the results presented here are based on an isotropic angular dependence of the inscattering profile (12) corresponding to $P_{0}(x)=1$. In the special case that the angular distribution is assumed to be $P_{1}(x)=x$, see, e.g., Ref. [38], the commonly used boundary condition (24) yields exact expressions for the odd expansion coefficients.

The influence of the approximation order of the Legendre expansion (3) is presented in Fig. 5. It can be seen, that the commonly used two-term approximation is not suitable to describe the prescribed distribution function $f^{S}(U, x)$ at the cathode. Hence, approximately 20 terms are required to obtain good numerical results at 


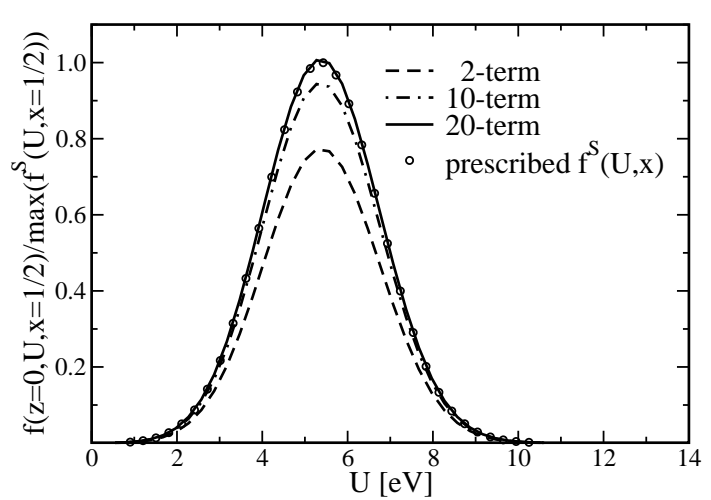

Fig. 5. Distribution functions $f(0, U, x)$ obtained by different orders of the Legendre expansion (3) using the boundary conditions (12) with the inscattering profile (22) of the electrons at the angular variable $x=1 / 2$.

the cathode-sided boundary and the effect of the higher order terms has to be included at the boundaries. At the same time, less than about ten terms have been found to be sufficient in general to yield convergent results in the plasma volume $[22,34,39]$.

The results presented have been obtained by prescribing an isotropic influx of electrons into the half-range $x=p_{z} / p$ with $0 \leq x \leq 1$ using the function (22), which results from the impact of e.g. ions, fast neutrals or photons impinging onto the cathode. Electrons, which diffuse back to the cathode e.g. after collisions and strike the electrode, are assumed to be totally reflected and to produce no further electrons at the cathode. An extension of the current approach to this more general case is immediately possible by modifying the source function (22) to include the secondary electron emission coefficients and different energy profiles for the different species as well as by combining the resulting influx conditions with the corresponding energy-dependent reflection conditions at $z=0$

$$
\begin{aligned}
& -\frac{1-r}{1+r} \sum_{i=0}^{\lfloor(l-1) / 2\rfloor} \frac{(2 \varrho+1) ! f_{2 i}(0, U)}{(2 \varrho-2 i+1) ! !(2 \varrho+2 i+2) ! !} \\
& =\sum_{i=0}^{\varrho} \frac{(2 \varrho+1) ! f_{2 i+1}(0, U)}{(2 \varrho-2 i) ! !(2 \varrho+2 i+3) ! !}
\end{aligned}
$$

for $\varrho=0, \ldots,\lfloor(l-1) / 2\rfloor$. Note that these relations differ from equations (17) by the minus sign on the left-hand side of (25) only.

\section{Summary}

The kinetic equation of the electrons is frequently treated by using an expansion of the electron momentum distribution function (EMDF) in Legendre polynomials. This approach leads to a system of partial differential equations whose numerical solution requires suitable boundary conditions. Based on relations for the macroscopic properties of the electrons and a fundamental integral relation of Legendre polynomials using a half-range integration technique in the angular space, relations between the expansion coefficients of the EMDF with an odd index and the expansion coefficients with an even index have been derived. These energy-dependent relations can be used as boundary conditions for spatially one-dimensional discharge configurations with plane-parallel electrodes. In particular, the new boundary conditions at the cathode yield a numerical solution of the electron kinetic equation which almost matches the prescribed profile. It has be shown that an improvement of numerical solutions can be achieved at a slightly higher computational cost when using Eq. (12). The approach presented makes it possible to treat correctly the 
influx of electrons due to, e.g., ion impact onto the cathode or thermionic emission as well as the partial reflection of electrons at the wall on the microscopic level. Its extension to obtain boundary conditions for arbitrary moments of the kinetic equation is immediately possible.

This work was supported by the Deutsche Forschungsgemeinschaft within the SFB TR 24.

\section{Appendix}

The well known double factorial notation

$$
n ! != \begin{cases}n \cdot(n-2) \cdot \ldots \cdot 3 \cdot 1 & \text { for } n>0 \text { odd } \\ n \cdot(n-2) \cdot \ldots \cdot 4 \cdot 2 & \text { for } n>0 \text { even } \\ 1 & \text { for } n \in\{0,-1\}\end{cases}
$$

can be extended to negative integers using the relations $[40]$

$$
\begin{aligned}
(-(2 n+1)) ! ! & =\frac{(-1)^{n}}{(2 n-1) ! !} \\
(-(2 n)) ! ! & =\infty
\end{aligned}
$$

for $n \in \mathbb{N}$. Eq. (28) is a direct consequence of the general relation $(2 n) ! !=2^{n} n$ ! with $n !=\infty$ for $n<-1$ [40]. With the definitions (26)-(28) and $n / \infty=0$ for every $-\infty<n<+\infty$, the fundamental integral relation

$$
\int_{0}^{1} d x x^{k} P_{n}(x)=\frac{k !}{(k-n) ! !(k+n+1) ! !}
$$

for the Legendre polynomials holds for arbitrary $k, n \in$ $\mathbb{N}_{0}$. Obviously, Eq. (29) is fulfilled for $n \in\{0,1\}$ and any $k \in \mathbb{N}_{0}$. Its general validity is proven by induction. Using the recurrence formula [40]

$$
P_{n+1}(x)=\frac{2 n+1}{n+1} x P_{n}(x)-\frac{n}{n+1} P_{n-1}(x)
$$

for the Legendre polynomials with $n \geq 1$, it follows that

$$
\begin{aligned}
\int_{0}^{1} d x & x^{k} P_{n+1}(x) \\
= & \int_{0}^{1} d x x^{k}\left[\frac{2 n+1}{n+1} x P_{n}(x)-\frac{n}{n+1} P_{n-1}(x)\right] \\
= & \frac{2 n+1}{n+1} \frac{(k+1) !}{(k-n+1) ! !(k+n+2) ! !} \\
& -\frac{n}{n+1} \frac{k !}{(k-n+1) ! !(k+n) ! !} \\
= & \frac{k !}{(k-(n+1)) ! !(k+(n+1)+1) ! !} .
\end{aligned}
$$

In the final step, the equation $n ! !=n \cdot(n-2) ! !$ has been used. It even holds for the double factorial of negative integer arguments.

Note that Eq. (29) provides a much simpler expression for the integral at the left-hand side than the well known solution of this integral in terms of the Gamma function [41]. Furthermore, notice that the relation

$$
\int_{-1}^{0} d x x^{k} P_{n}(x)=(-1)^{k+n} \int_{0}^{1} d x x^{k} P_{n}(x)
$$

holds for $k, n \in \mathbb{N}$.

\section{References}

1. I.P. Shkarofsky, T.W. Johnston, M.P. Bachynski, The Particle Kinetics of Plasmas (Addision-Wesley Publishing Company, Montreal, Canada, 1966)

2. R. Winkler, J. Wilhelm, V. Schüller, Contrib. Plasma Phys. 10, 51 (1970)

3. R.E. Robson, K.F. Ness, Phys. Rev. A 33, 2068 (1986)

4. D. Loffhagen, F. Sigeneger, R. Winkler, in Low Temperature Plasmas: Fundamentals, Technologies and Techniques, edited by R. Hippler, H. Kersten, M. Schmidt, 
K.H. Schoenbach (WILEY-VCH, Weinheim, 2008), Vol. 1, chap. 2, pp. 15-45, 2nd edn.

5. Y.I. Matveenko, D.A. Gryaznykh, A.A. Kondrat'ev, I.A. Litvinenko, J. Phys. A: Math. General 41, 355501 (2008)

6. G.L. Braglia, J.J. Lowke, J. Phys. D: Appl. Phys. 12, 1831 (1979)

7. J.P. Boeuf, E. Marode, J. Phys. D: Appl. Phys. 15, 2169 (1982)

8. V.D. Stojanovic, Z.L. Petrovic, J. Phys. D: Appl. Phys. 31, $834(1998)$

9. S. Longo, Plasma Sources Sci. Technol. 9, 468 (2000)

10. W. Yu, L.Z. Zhang, J.L. Wang, L. Han, G.S. Fu, J. Phys. D: Appl. Phys. 34, 3349 (2001)

11. S. Dujko, R.D. White, Z.L. Petrovi, J. Phys. D: Appl. Phys. 41, 245205 (2008)

12. C. Busch, U. Kortshagen, Phys. Rev. E 51, 280 (1995)

13. U. Kortshagen, C. Busch, L.D. Tsendin, Plasma Sources Sci. Technol. 5, 1 (1996)

14. L.L. Alves, G. Gousset, C.M. Ferreira, Phys. Rev. E 55, $890(1997)$

15. D. Loffhagen, F. Sigeneger, Plasma Sources Sci. Technol. 18, $034006(2009)$

16. D. Uhrlandt, R. Winkler, J. Phys. D: Appl. Phys. 29, 115 (1996)

17. S. Arndt, D. Uhrlandt, R. Winkler, Plasma Chem. Plasma Process. 21, 175 (2001)

18. S. Arndt, D. Uhrlandt, R. Winkler, J. Phys. D: Appl. Phys. 34, $1982(2001)$

19. D. Loffhagen, R. Winkler, J. Phys. D: Appl. Phys. 34, 1355 (2001)

20. S. Arndt, F. Sigeneger, R. Winkler, Plasma Chem. Plasma Process. 23, 439 (2003)
21. F. Sigeneger, D. Loffhagen, IEEE Trans. Plasma Sci. 35 $1260(2007)$

22. D. Loffhagen, F. Sigeneger, R. Winkler, J. Phys. D: Appl. Phys. 35, 1768 (2002)

23. J.J. Lowke, J.H. Parker, C.A. Hall, Phys. Rev. A 15, 1237 (1977)

24. T. Petrova, G.M. Petrov, Physica Scripta 61, 102 (2000)

25. B. Li, R.D. White, R.E. Robson, J. Phys. D: Appl. Phys. 35, $2914(2002)$

26. G. Petrov, R. Winkler, Plasma Chem. Plasma Process. 18, $113(1998)$

27. M. Hannemann, P. Hardt, D. Loffhagen, M. Schmidt, R. Winkler, Plasma Sources Sci. Technol. 9, 387 (2000)

28. D. Loffhagen, R. Winkler, Z. Donkó, Eur. Phys. J. AP 18, $189(2002)$

29. F. Sigeneger, R. Winkler, Plasma Chem. Plasma Process. 25, $147(2005)$

30. R.E. Marshak, Phys. Rev. 71, 443 (1947)

31. R.E. Robson, Aust. J. Phys. 34, 223 (1981)

32. J.P. England, H.R. Skullerud, Aust. J. Phys. 50, 553 (1997)

33. I.A. Porokhova, Y.B. Golubovskii, J.F. Behnke, Phys. Rev. E 71, 066406 (2005)

34. G.K. Grubert, D. Loffhagen, IEEE Trans. Plasma Sci. 35, $1215(2007)$

35. G. Petrov, R. Winkler, J. Phys. D: Appl. Phys. 30, 53 (1997)

36. R. Robson, Introductory transport theory for charged particles in gases (World Scientific Publishing Co. Pte. Ltd., Singapore, 2006)

37. P.J. Chantry, J. Appl. Phys. 62, 1141 (1987) 
38. T. Koshikawa, R. Shimizu, J. Phys. D: Appl. Phys. 7, 1303

$(1974)$

39. R.D. White, R.E. Robson, B. Schmidt, M.A. Morrison, J.

Phys. D: Appl. Phys. 36, 3125 (2003)

40. E.W. Weisstein, CRC Concise Encyclopedia of Mathematics (CRC Press, 1999)

41. I. Gradshteyn, I. Ryzhik, A. Jeffrey, Table of integrals, series, and products (Academic Press, INC., London, 1980) 